\title{
As Consequências da Distribuição Aleatória da Energia
}

$O$ presente artigo pretende apresentar uma estratégia muito importante para a aprendizagem das consequências do $2^{\circ}$ Princípio da Termodinâmica, chamada de «baralhamento de quanta" ( quantum shuffling") e apoiada num modelo pedagógico supersimplificado do estado sólido designado por sólido de Einstein. Tal estratégia permite nâo só a compreensão e operacionalização do equilíbrio térmico como também a caracterização da distribuiçâo da energia na matéria, a definição microscópica de temperatura e a harmonização desta com a respectiva definiçâo dentro da termodinâmica clássica (macroscópica). Todas estas conclusões perfeitamente gerais e válidas e com um valor explicativo muito grande, sāo feitas com base num modelo, repita-se, supersimplificado apontando-se num último ponto as correcçôes que deverão ser feitas para os casos reais.

\section{Introduçāo: proposta de uso de um modelo}

Os esquemas de probabilidade e de análise combinatória, essenciais para uma modelização cabal, embora simplificada das consequências do $2^{\underline{9}}$ princípio, estando ausentes da fase operacional concreta piagetiana de desenvolvimento, levam à adopção de estratégias alternativas numa fase inicial do curriculum (Ogborn, 1988), para incorporar, essencialmente, as ideias de irreversibilidade (distinguindo-a de irretornabilidade), de sequência natural dos fenómenos, de tendência para a uniformidade (Solomon, 1982) e, ainda e finalmente, para a ideia de espalhamento aleatório da energia; já portadores destas ideias os alunos seriam confrontados com a estratégia a desenvolver neste artigo (vide alternativas em Costa Pereira, 1986) e que se supõe adequada à fase terminal do ensino secundário (Stokes, 1984).

A «descoberta» da quantização da energia electrónica empreendida por uma via de descoberta guiada, comum a quase todos os modernos curricula secundários de Química, quer seja baseada na medida das energias de ionização ou na observação dos espectros, leva a reflectir sobre a distribuição da energia na matéria. Considerando a energia disponível às temperaturas correntes, pode-se ser levado à conclusão que essencialmente a maior aleatoriedade, dados os substanciais espaçamentos entre os níveis quânticos, se consegue distribuindo toda a energia pelo máximo número possível de entidades, correspondendo este estado de coisas, na prática, à verificação absolutamente contingental do, tantas vezes indevidamente, generalizado princípio de energia mínima. Bent (Bent, 1965) apresenta, no entanto, um conceito muito importante que é o da temperatura para a qual estão identicamente povoados o primeiro e segundo níveis quânticos. Verifica-se que essa temperatura, tipicamente, para o caso da energia electrónica, é de $40000 \mathrm{~K}$, o que, confirmando as nossas previsões, invalida o princípio de «Aufbau» para temperaturas como as solares, onde um número razoável de configurações excitadas devem existir.

Pode generalizar-se a ideia de quantização à energia associada aos outros movimentos das partículas, sendo os alunos levados a admitir, desta feita, temperaturas para a igualdade de populações entre os dois primeiros níveis quânticos (Bent, 1965) da ordem de $10^{-17} \mathrm{~K}$ para a translacção, de $2 \mathrm{~K}$ para a rotação e de $2000 \mathrm{~K}$ para a vibração (note-se, a título de curiosidade, que, para a energia nuclear, é apontado um valor de $10^{11} \mathrm{~K}$ ). Assim sendo, é fácil compreender-se que os estados quânticos de translacção representam praticamente um contínuo, e que, para as kemperaturas normais à superfície da Terra, não há restrições impostas pela quantização da rotação. Outro tanto já não se passa com a vibração, podendo dizer-se, de uma forma geral, que um número razoável de configurações excitadas devem existir. Para se estudar esse problema, isto é as consequências da aleatoriedade na distribuição da energia pela matéria, ainda nos anos setenta, o grupo do Prof. Black (Black \& al, 1971) teve a ideia de recorrer a um modelo supersimplificado de um sólido, (no fundo um conjunto de osciladores harmónicos, independentes entre si e que podem estar em estados energéticos correspondentes a 0 ou a um número inteiro dos mesmos quanta de energia) chamado sólido de Einstein, e sobre o qual simulou aquilo a que chamou o «baralhamento dos quanta» («quantum shuffling»). Note-se que nos sólidos reais há sempre uma energia residual de vibração e os espaçamentos não são iguais.

Tal exercício pode ser feito com a ajuda de dois dados, cujos lançamentos aleatórios condicionam a movimentação, numa grelha de $6 \times 6$, de marcas simbolizando os quanta de energia. Uma concretização de tal modelo pode ser feita para o caso de 36 «átomos» por um tabuleiro como o da Figura 1, em que os quanta são simbolizados por marcas e os «átomos» pelas casas. As movimentações são feitas de acordo com os lançamentos de dois dados (um referindo-se ao $\mathrm{n}^{\mathrm{o}}$ da linha e outro à coluna). $\mathrm{O}$ primeiro lançamento dos dois dados define a casa de partida, se contiver alguma marca, definindo o segundo lançamento a casa de chegada.

\footnotetext{
Faculdade de Ciências da Universidade do Porto.
} 


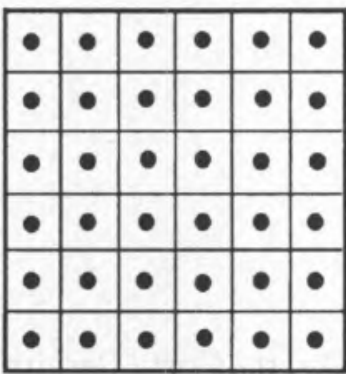

A-no início

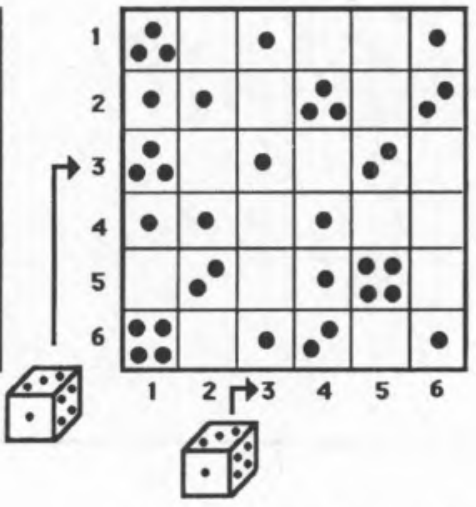

B-após alguns "lançamentos"
FIGURA 1

Modelo do sólido de Einstein para 36 osciladores

Ainda melhor, para se atingirem algumas das conclusões quantitativas que se pretendem, será através de um programa de computador, disponível comercialmente e também muito fácil de implementar, que essencialmente replica o que acontece no tabuleiro mas para muito mais átomos e muito mais rapidamente (vide Figura 2A, B e C).

Primeira constatação: existência do equilíbrio térmico

O primeiro aspecto a fazer ressaltar do exercício de «baralhamento de quanta» é o facto de, a partir de uma determinada altura, se atingir uma configuração razoavelmente estável (em termos que excluam a identidade das casas ocupadas) e que, se o número de casas for suficientemente grande, o número relativo das que contêm 0 quanta (note-se que isto não acontece nos vibradores atómicos) e os múltiplos inteiros (note-se que isto também não acontece nos vibradores atómicos) do quantum de vibração, permanece relativamente estável, muito embora as «trocas» a nível «microscópico» se continuem a dar. Com efeito, começando-se por um estado inicial de um quantum por oscilador (Figura 1A ou 2A), ao fim de algum tempo atingem-se configurações mais prováveis (Figura $1 \mathrm{~B}$ ou $2 \mathrm{C})$, verificando-se que a probabilidade das configu-

\begin{tabular}{|c|c|c|c|c|c|}
\hline 5 & Shuffle & 0 & A & Auerage & $\begin{array}{l}\text { OFF } \\
\text { ON }\end{array}$ \\
\hline 0 & Quit & & $\mathbf{P}$ & Probability & $\begin{array}{l}\text { OFF } \\
\text { ON }\end{array}$ \\
\hline
\end{tabular}

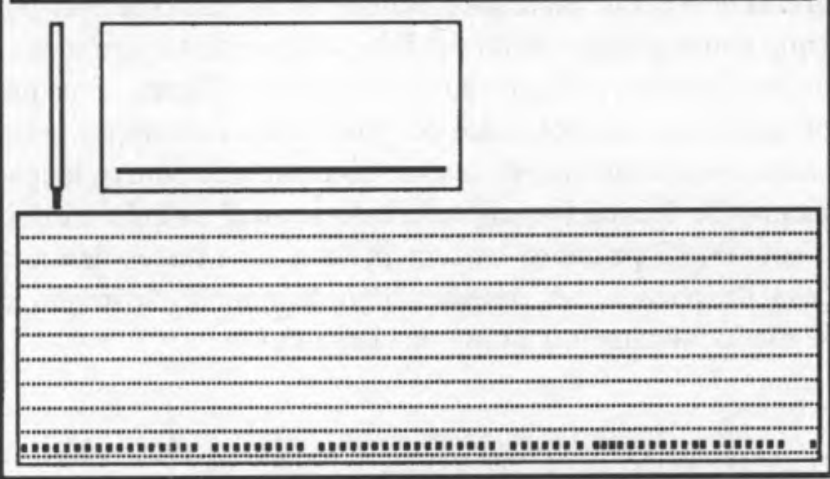

B-Depois de algum tempo

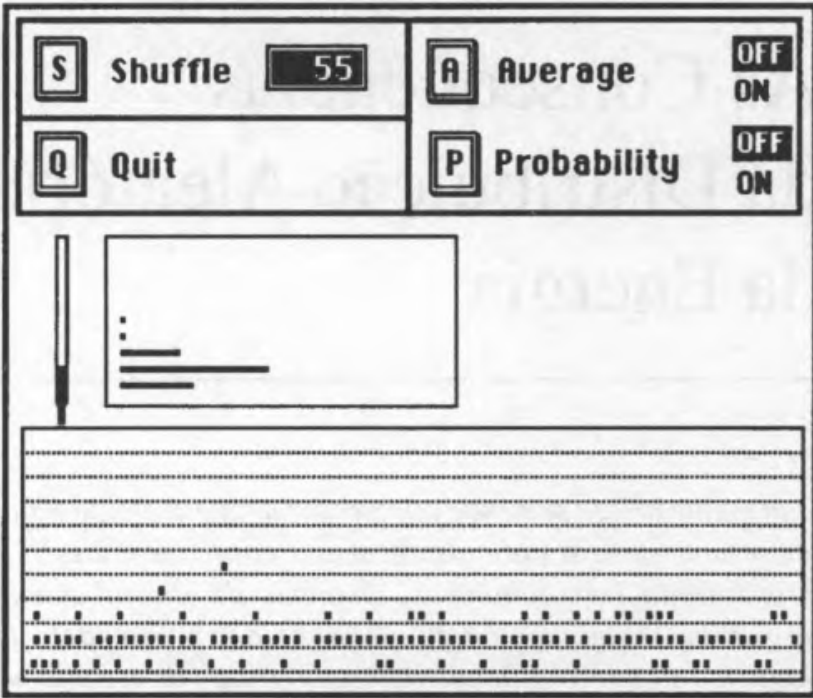

C-Mais tarde

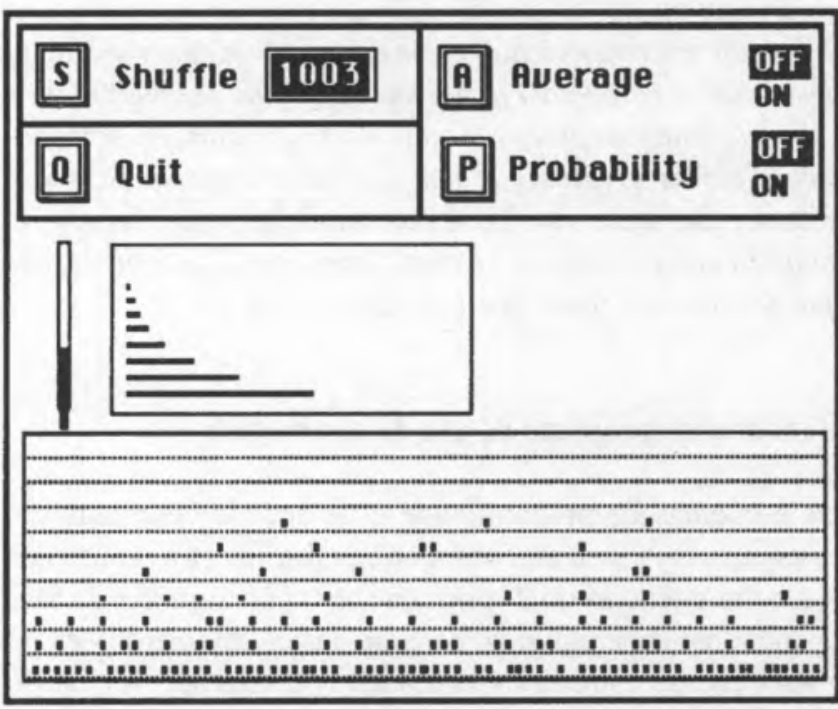

FIGURA 2

Modelo do Sólido de Einstein, Baralhamento de quanta em 200 partículas

rações (proporcional ao $\mathrm{n}^{0}$ de realizações diferentes possíveis - microestados - destas: W), embora se continuem a processar as transformações a nível «atómico», estabiliza razoavelmente (Figura 3).

\begin{tabular}{|c|r|r|r|r|r|r|r|c|}
\hline $\begin{array}{c}\text { Número de } \\
\text { lançamentos }\end{array}$ & $n_{0}$ & $n_{1}$ & $n_{2}$ & $n_{3}$ & $n_{4}$ & $n_{5}$ & $n_{6}$ & $\begin{array}{c}W \\
\text { (Probabilidade) }\end{array}$ \\
\hline 5 & 5 & 26 & 5 & & & & & $6 \times 10^{10}$ \\
\hline 10 & 7 & 22 & 7 & & & & & $1 \times 10^{14}$ \\
\hline 20 & 13 & 12 & 9 & 2 & & & & $2 \times 10^{17}$ \\
\hline 30 & 17 & 8 & 5 & 6 & & & & $3 \times 10^{17}$ \\
\hline 40 & 16 & 11 & 2 & 7 & & & & $1 \times 10^{17}$ \\
\hline 50 & 17 & 11 & 3 & 2 & 2 & 1 & & $1 \times 10^{18}$ \\
\hline 60 & 16 & 13 & 2 & 2 & 2 & 1 & & $4 \times 10^{17}$ \\
\hline 70 & 20 & 8 & 2 & 2 & 2 & 2 & & $2 \times 10^{17}$ \\
\hline 80 & 18 & 10 & 2 & 3 & 2 & 1 & & $7 \times 10^{17}$ \\
\hline 90 & 20 & 8 & 3 & 1 & 1 & 3 & & $1 \times 10^{17}$ \\
\hline 100 & 19 & 9 & 3 & 2 & 1 & 1 & 1 & $7 \times 10^{17}$ \\
\hline
\end{tabular}

Evolução das configuraçōes do sólido de Einstein (exemplo manual de 36 osciladores) 
É exactamente neste facto que consiste o equilíbrio térmico, que assim fica perfeitamente simulado. Ele não é mais que a tendência para as configurações mais prováveis observada quando da distribuição da energia pela matéria.

Segunda constatação: constância da razão do nível de ocupação de estados e significado físico desta constante

Seja qual for o exercício de baralhamento de quanta por que se tenha optado, interessa chamar a atenção para a constância (aproximada) da razão - r - entre as populações de níveis vizinhos e encontrar o significado físico dessa razão. Claro está que a constância será muito mais rigorosa na versão computador (Figura 2) do que na versão manual (Figura 1). Quanto ao significado físico dessa razão basta considerar que o número total de partículas $\mathrm{N}$ deve ser dado pela soma das que têm zero quanta $\left(\mathrm{n}_{0}\right)$, mais das que têm um quantum $\left(\mathrm{n}_{1}\right)$, mais das que têm dois quanta $\left(\mathrm{n}_{2}\right)$, mais das que têm três quanta $\left(\mathrm{n}_{3}\right)$, etc.

$\mathrm{n}_{0}+\mathrm{n}_{1}+\mathrm{n}_{2}+\mathrm{n}_{3}+\ldots=\mathrm{N}$

enquanto que o número de quanta total q, deverá ser dado pela soma:

$\mathrm{n}_{1}+2 \mathrm{n}_{2}+3 \mathrm{n}_{3}+\ldots=\mathrm{q}$

Atendendo à verificada constância da razão $r$ entre as populações de níveis vizinhos, tem-se:

$\mathrm{r}=\mathrm{n}_{0} / \mathrm{n}_{1}=\mathrm{n}_{1} / \mathrm{n}_{2}=\mathrm{n}_{2} / \mathrm{n}_{3}=\ldots>1$

e

$r^{\prime}=1 / r<1$

Por substituição em (1) e (2), obtêm-se as somas:

$\mathrm{S}=1+\mathrm{r}^{\prime}+\mathrm{r}^{\prime 2}+\mathrm{r}^{13}+\ldots=\mathrm{N} / \mathrm{n}_{0}$

$\mathrm{T}=\mathrm{r}^{\prime}+2 \mathrm{r}^{\prime 2}+3 \mathrm{r}^{\prime 3}+\ldots=\mathrm{q} / \mathrm{n}_{0}$

Para um grande número de partículas e quanta é legítimo tomar a soma infinita da série, que para estes casos bem conhecidos (já que $1 / \mathrm{r}<1$ ) é respectivamente:

$\mathrm{S}=1 /\left(1-\mathrm{r}^{\prime}\right)=\mathrm{N} / \mathrm{n}_{0}$

$\mathrm{T}=\mathrm{r}^{\prime} /\left(1-\mathrm{r}^{\prime}\right)^{2}=\mathrm{q} / \mathrm{n}_{0}$

Ou então

$\mathrm{r}=1+\mathrm{N} / \mathrm{q}$

Por outro lado da expressão facilmente deduzível do número de microestados do sistema

$\mathrm{W}=(\mathrm{N}-1+\mathrm{q}) ! /(\mathrm{N}-1) ! \mathrm{q} !$ quando se fornece um quantum de energia ao sistema, o número de microestados será:

$\mathrm{W}^{\prime}=(\mathrm{N}+\mathrm{q}) ! /(\mathrm{N}-1) !(\mathrm{q}+1) !$

Da comparação directa de (4) e (5), resulta atendendo a que $\mathrm{q}+1 \sim \mathrm{q}$

$\mathrm{W}^{\prime}=\mathrm{W}(1+\mathrm{N} / \mathrm{q})$

e atendendo a (3), vem finalmente:

$\mathrm{W}^{\prime}=\mathrm{rW}$

O que acarreta para r o significado físico de o número por que resulta multiplicado o número de microestados do sistema, quando a ele se adiciona um quantum.

\section{Terceira constatação: caracterização dos estados térmi- cos dos sistemas e do equilíbrio térmico}

A razão $\mathrm{r}$, ou melhor $1 / \mathrm{r}$, aparece como caracterizando os estados térmicos dos sistemas e a explicar factos como o de os corpos frios aquecerem e o de os corpos quentes arrefecerem, quando postos em contacto. Com efeito, atendendo ao significado físico de $r$ descrito no número anterior, a redução do número de microestados operada pela extraç̧ão de um quantum no corpo quente ( $1 / \mathrm{r}$ maior) é sempre menor que o aumento do número de microestados correspondente à aquisição de um quantum pelo corpo frio, por isso (por ser no sentido da maior probabilidade) é que maioritariamente e portanto inexoravelmente na escala macroscópica a passagem dos quanta se faz dos corpos quentes para os frios, convergindo os valores de $\mathrm{r}$ dos dois corpos para um $\mathrm{r}$ intermédio. Podem efectuar-se simulações por computador (Figura 4) que permitem a confirmação desta forma de enunciar o $2^{\circ}$ Princípio da Termodinâmica.

A necessidade de usar medidas mais manejáveis e aditivas (em vez de multiplicativas como as probabilidades de acontecimentos independentes e consequentemente o número de microestados são) leva à contabilização dos microestados através da função de estado $S$, chamada entropia e em cuja definição intervém k, constante de Boltzman, já introduzida no curriculum com o significado de constante dos gases perfeitos por uma molécula:

$\mathrm{S}=\mathrm{k} \ln \mathrm{W}$

O equilíbrio acontece por maximização da entropia do universo, ou quando

$\Delta \mathrm{S}_{\text {universo }}=\Delta \mathrm{S}_{\text {sistema }}+\Delta \mathrm{S}_{\text {exterior }}$

é igual a zero. 
A-Os dois corpos isolados

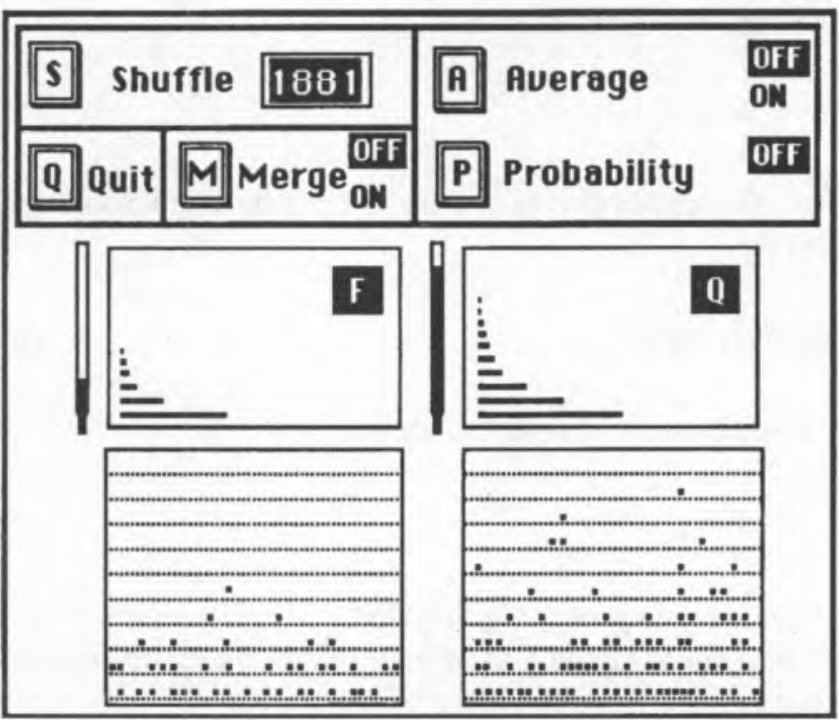

B-Depois de postos em contacto

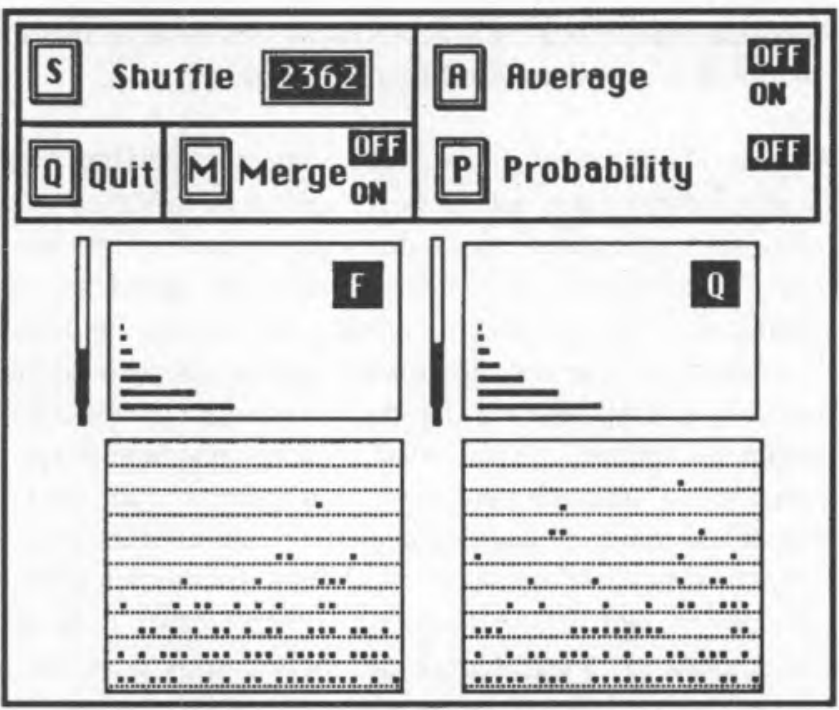

FIGURA 4

Modelo do sólido de Einstein - Equilíbrio térmico

Quarta constatação: definição microscópica de temperatura e coincidência desta com a definição macroscópica

Utilizando a função entropia para caracterizar a transferência de um só quantum ter-se-á, para a variação de entropia associada a essa transferência, atendendo a (7) e (8):

\section{$\Delta \mathrm{S}=\mathrm{k} \ln \mathrm{W}^{\prime}-\mathrm{k} \ln \mathrm{W}=\mathrm{k} \ln \mathrm{r}$}

Considerando agora não a transferência de um quantum mas de uma quantidade de energia que vai provocar um aumento $\mathrm{dU}$ da energia interna do sistema, correspondendo a $\mathrm{dU} / \varepsilon$ quanta, se for $\varepsilon$ a energia de cada quantum, ter-se-á pois (já que para uma quantidade infinitesimal de variação de energia interna $\mathrm{r}$ não variará apreciavelmente):
Se se tomar como medida do estado térmico do sistema, em vez de $1 / r, T=\varepsilon / k \ln r$ (note-se que então será r=e-e/kT) obtémse uma medida ideal para o caracterizar, pois que para além de variar monótona e inversamente com $\mathrm{r}$, em vez de variar como $1 / \mathrm{r}$ entre 0 e 1 , varia entre 0 e $\infty$. Esta quantidade que depende dos espaçamentos dos níveis quânticos dos sistemas, designa-se por temperatura e a ela corresponde, como se pode ver por substituição a partir de (11) a definição macroscópica da Termodinâmica Clássica no caso do volume e do número de partículas não variarem.

$\mathrm{T}=\mathrm{dU} / \mathrm{dS}$

Conclusão: de como um modelo muito grosseiro permite conclusões muito válidas

A estratégia exposta permite a compreensão de conceitos difíceis como o de equilíbrio térmico, entropia e temperatura com toda a naturalidade e operacionalidade. Permite também, o que é muitíssimo importante, fazer a ligação entre o macroscópico e o microscópico como se viu na caracterização do equilíbrio térmico, que não exclui a movimentação a nível microscópico e a caracterização do conceito de temperatura tanto a nível microscópico como macroscópico. Tanto a temperatura como o equilíbrio são conceitos que se aplicam em condições muito diferentes das do modelo devendo referir-se que aquela, tendo em conta o espaçamento variável entre os estados quânticos, é uma verdadeira medida do estado térmico do sistema e que a ideia de equilíbrio se pode estender a fenómenos muito mais complicados, como quando ocorrem reacções químicas, mantendo-se essencialmente a mesma conceptualização: maximização da entropia. As grandes diferenças do modelo relativamente às condições reais dos sólidos, são, como foi dito, o facto de nestes a energia do estado fundamental ser diferente de 0 e de o espaçamento entre níveis quânticos não ser uniforme. Isto traduz-se, na prática, por a função de distribuição da energia conter um termo quântico - $\mathrm{f}(\mathrm{E})$ - para além do conhecido e celebrado termo estatístico, o factor de Boltzman - e-E/kT (derivável da nova definição de $\mathrm{r}$, como $\mathrm{r}=\mathrm{e}^{\ell / \mathrm{kT}}$ ) e que representa o número de osciladores com uma energia superior à energia $\mathrm{E}$ :

$N(d E)=f(E) \cdot e^{-E / K T} d E$

Tal conduz a uma distribuição diferente (Figura 6) (não rigorosamente exponencial) da energia relativamente ao que acontecia no sólido de Einstein (Figura 5), mas não invalida a grande utilidade, pelo menos pedagógica, do modelo. Com efeito, verificou-se que com toda a naturalidade, surgem os fundamentos do equilíbrio químico (no equilíbrio térmico) e da cinética química (no factor de Boltzman). Mais espantoso ainda é o facto de os resultados obtidos sobre $\mathrm{T}$ e $\mathrm{S}$ serem absolutamente gerais. 


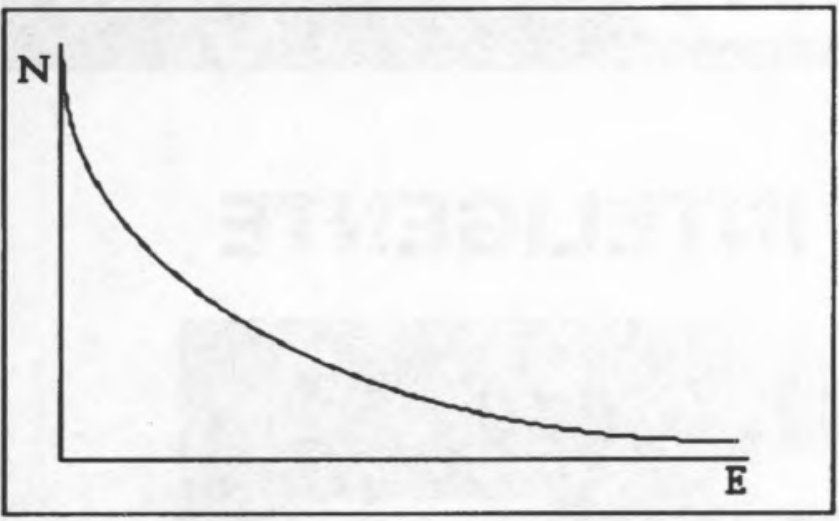

FIGURA 5

Distribuiçâo da energia no sólido de Einstein

Referências

- Bent H. A., The Second Law, Oxford University Press, New York, 1965 - Black P. J., Davies P., Ogborn J., A Quantum Shuffling Game for Teaching Statistical Mechanics, Am. J. Phys., vol 39, 1154, 1971

- Costa Pereira D., O Conceito de Entropia e o Ensino da Química, Boletim da SPQ, 25, 25-32, Lisboa, 1986

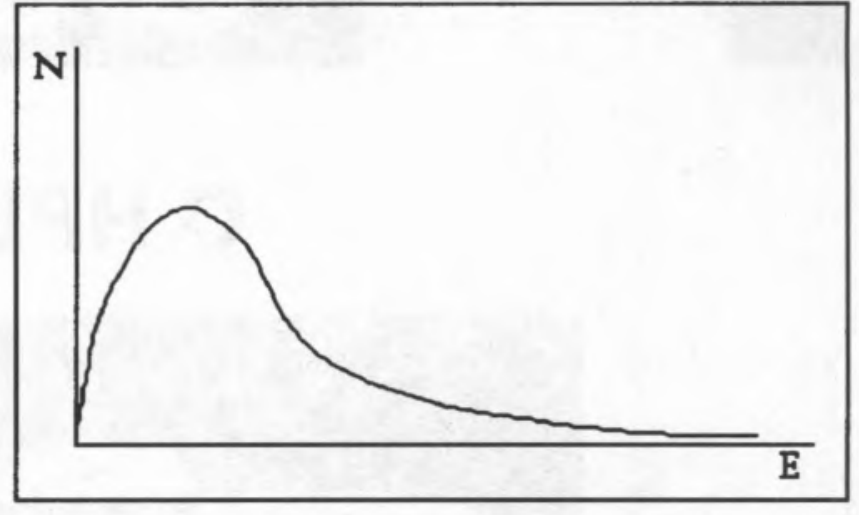

FIGURA 6

Distribuiçâo da energia num sólido real

- Ogborn J., The Nature of Science and its Implications in Science For All, Actas do I Encontro sobre Educação em Ciêțias, 107-124, Braga, 1988

- Solomon J., How Children Learn about Energy or Does the First Law Come First?, School Science Review, Março, 415-422, 1982

-Stokes B. J. (ed), Nuffield Advanced Chemistry (Revised), Teachers Guide, vol I \& II, Longman, Harlow, 1984

\section{WALTHER NERNST (1864-1941)}

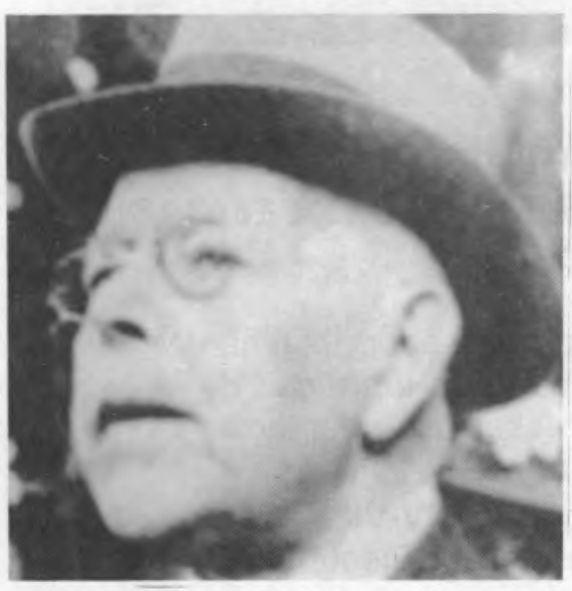

Nernst nasceu em Brieson, na Prússia (hoje, Wabrzezno, Polónia) e estudou na Universidade de Würzburg onde obteve o seu doutoramento em 1887. Nesse mesmo ano foi trabalhar com Ostwald em Leipzig como assistente de investigação e em 1891 tornou-se professor de Química-Física na Universidade de Göttingen. Em 1905 foi nomeado professor de Química-Física na Universidade de Berlim e em 1920 recebeu o Prémio Nobel da Química.

Nernst deixou muitas contribuições preciosas nos domínios da Física e da Química-Física. Aplicou os princípios da Termodinâmica às células electroquímicas e chegou a uma expressão para o potencial eléctrico em função das concentrações. Interpretou o comportamento dos iões em solução aquosa. Em 1905 tratou teoricamente o problema da entropia das substâncias na vizinhança do zero absoluto; esta grande contribuição para a ciência é hoje conhecida como o «teorema do calor de Nernst» ou a «terceira lei da termodinâmica». Em termos cinéticos, Nernst explicou a combinação fotoquímica do hidrogénio e do cloro através de uma cadeia de reacções envolvendo os átomos destes elementos. Anteriormente, Max Bodenstein (1871-1942) tinha tentado explicar a mesma reacção recorrendo a iões e moléculas excitadas sem resultado satisfatório. A explicação de Nernst conduziu a importantes desenvolvimentos na Cinética Química das reacções em cadeia. 


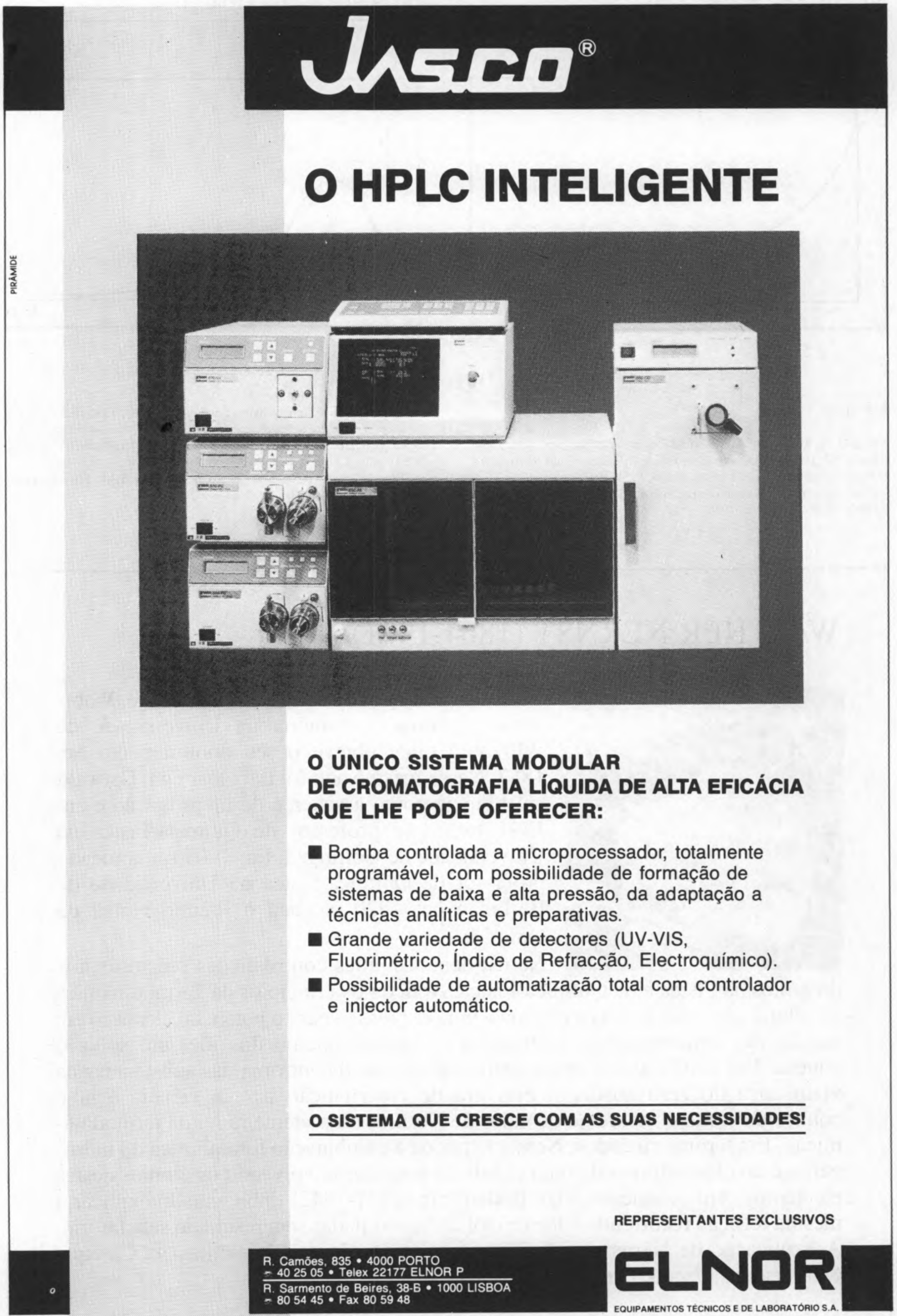

